\title{
MAXWELL EQUATIONS FOR A GENERALISED LAGRANGIAN FUNCTIONAL
}

\section{ECUACIONES DE MAXWELL PARA UNA FUNCIONAL DE LAGRANGE GENERALIZADA}

\author{
H. Torres-Silva ${ }^{1}$ \\ Recibido el 5 de septiembre de 2007, aceptado el 29 de noviembre de 2007 \\ Received: September 5, $2007 \quad$ Accepted: November 29, 2007
}

\begin{abstract}
RESUMEN
En este trabajo se aborda el problema de la construcción de la funcional de Lagrange de un campo electromagnético. Se introducen las ecuaciones generalizadas de Maxwell de un campo electromagnético en el espacio libre. La idea principal se basa en el cambio de función de Lagrange en virtud de la acción integral. Por lo general, la funcional de Lagrange, que describe el campo electromagnético, se construye con el cuadrado del tensor de campo electromagnético. Ese término cuadrático es la razón, desde un punto de vista matemático, de la forma lineal de las ecuaciones de Maxwell en el espacio libre. Se obtienen las ecuaciones no lineales de Maxwell sin considerar esta suposición. Las ecuaciones obtenidas son bastante similares a las conocidas ecuaciones de Maxwell. Se analiza el tensor de energía del campo electromagnético en un enfoque quiral de la Lagrangiana de Born Infeld en relación con la constante cosmológica.
\end{abstract}

Palabras clave: Lagrange, acción, ecuaciones de Maxwell, Born Infeld.

\section{ABSTRACT}

This work deals with the problem of the construction of the Lagrange functional for an electromagnetic field. The generalised Maxwell equations for an electromagnetic field in free space are introduced. The main idea relies on the change of Lagrange function under the integral action. Usually, the Lagrange functional which describes the electromagnetic field is built with the quadrate of the electromagnetic field tensor $F^{i k}$. Such a quadrate term is the reason, from a mathematical point of view, for the linear form of the Maxwell equations in free space. The author does not make this assumption and nonlinear Maxwell equations are obtained. New material parameters of free space are established. The equations obtained are quite similar to the well-known Maxwell equations. The energy tensor of the electromagnetic field from a chiral approach to the Born Infeld Lagrangian is discussed in connection with the cosmological constant.

Keywords: Lagrange, action, Maxwell equations, Born Infeld.

\section{INTRODUCTION}

The action integral (built to formulate the least-square principle [1]) for a process in an electromagnetic field has the following form:

$$
I=\int_{T} \int_{V}\left(\left(S_{v}\right)-(\rho V-\boldsymbol{j} \cdot \boldsymbol{A})-\frac{1}{2}\left(\mu_{0}^{-1} \boldsymbol{B}^{2}-\varepsilon_{0} \boldsymbol{E}^{2}\right)\right) d V d t
$$

All physical phenomena in the electromagnetic field take place so the action integral has the minimal value $\delta I=0$. The theory of the electromagnetic field [1] leads to the
Lagrange motion equations for an electric charge in the electromagnetic field and defines the electromagnetic field tensor:

$$
F_{i k}=\frac{\partial A_{k}}{\partial x^{i}}-\frac{\partial A_{i}}{\partial x^{k}}
$$

Eqns. (2) are equivalent to the first pair of Maxwell equations:

$$
\frac{\partial F_{i k}}{\partial x^{l}}+\frac{\partial F_{k l}}{\partial x^{i}}+\frac{\partial F_{l i}}{\partial x^{k}}=0 \quad \text { for } \quad i \neq k \neq l \neq i
$$

\footnotetext{
1 Instituto de Alta Investigación. Universidad de Tarapacá. Antofagasta No 1520. Arica, Chile. E-mail: htorres@uta.cl
} 
Or, in three-dimensional notation:

$$
\operatorname{curl} \boldsymbol{E}=-\dot{\boldsymbol{B}} \quad \text { and } \quad \operatorname{div} \boldsymbol{B}=0
$$

Making calculations of variation for the functional $I$ with respect to the four-dimensional potential $A^{i}$ one obtain the second pair of Maxwell equations:

$$
\frac{\partial F^{i k}}{\partial x^{k}}=-\mu_{0} j^{i}
$$

Or in equivalent vector notation:

$$
\operatorname{div}\left(\varepsilon_{0} \boldsymbol{E}\right)=\rho \text { and } \operatorname{curl}\left(\mu_{0}^{-1} \boldsymbol{B}\right)=\boldsymbol{j}+\varepsilon_{0} \dot{\boldsymbol{E}}
$$

From the mathematical point of view, the demand for a linear form of Maxwell equations for free space compels one to assume that the field term (the third in integral eqn. (1)) must be built with the electromagnetic field tensor $F^{i k}$ second power (the exponent of any power function under the action integral is one less after calculating the variation). So, the equations obtained are linear with respect to the electromagnetic field tensor $F^{i k}$. The action integral I could be rewritten in the following form:

$$
I=\int_{T} \int_{V}\left(S_{v}-(\rho V-\boldsymbol{j} \cdot \boldsymbol{A})-\Delta e_{V}\right) d V d t
$$

Where

$$
\Delta=\Delta e_{V}=\frac{1}{2} \mu_{0}^{-1} \boldsymbol{B}^{2}-\frac{1}{2} \varepsilon_{0} \boldsymbol{E}^{2}=\frac{1}{4} \mu_{0}^{-1} F^{i k} F_{i k}
$$

The linear form of the Maxwell equations, from the mathematical point of view, is arbitrarily assumed by eqn. (7). In addition the linear character of Maxwell equations for free space (as well as for air) has been confirmed by many experiments. There is no doubt that linear Maxwell equations, within experimental precision, are satisfied, however, we could not reject other mathematical forms of the electromagnetic field equations.

Is we assume, more generally, that the action integral is built with the help of a function $f(\cdot)$, it could be written:

$$
I=\int_{T} \int_{V}\left(S_{v}-(\rho V-j A)-f\left(\frac{1}{2} \mu_{0}^{-1} B^{2}-\frac{1}{2} \varepsilon_{0} E^{2}\right)\right) d V d t
$$

(the codomain for the function $f(\cdot)$ is the real set).

Under this assumption new Maxwell equations having the same mathematical structure are obtained. The wave speed in free space for these new Maxwell equations will also be equal to " $c$ ".

\section{MAXWELL EQUATIONS FOR THE GENERALISED FUNCTIONAL}

The second pair of Maxwell equations in the case of the generalised action integral eqn. (8) is obtained after evaluating the variation of the action with respect to the four-dimensional potential $A^{i}$. We can denote

$$
\begin{aligned}
\delta I & =\int_{T} \int_{V}-\left(j^{i} \delta A_{i}\right)-\delta f\left(\frac{1}{4} \mu_{0}^{-1} F^{i k} F_{i k}\right) d V d t \\
& =\int_{T} \int_{V}-\left(j^{i} \delta A_{i}\right)-f^{\prime}(\Delta)\left(\frac{1}{4} 2 \mu_{0}^{-1} F^{i k} \delta F_{i k}\right) d V d t
\end{aligned}
$$

According to eqn. (2) we could write

$$
\begin{aligned}
0 & =\delta I \\
& =-\int_{T} \int_{V}\left(j^{i} \delta A_{i}+f^{\prime}(\Delta)\right)\left(\frac{1}{2} \mu_{0}^{-1} F^{i k}\left(\frac{\partial \delta A_{k}}{\partial x^{i}}-\frac{\partial \delta A_{i}}{\partial x^{k}}\right)\right) d V d t
\end{aligned}
$$

After interchanging the indices ' $i$ ' and ' $k$ ' in the final term we obtain:

$$
\begin{aligned}
0 & =\int_{T} \int_{V}\left(j^{i} \delta A_{i}+f^{\prime}(\Delta)\right)\left(\frac{1}{2} \mu_{0}^{-1} F^{i k}\left(\frac{\partial \delta A_{k}}{\partial x^{i}}-F^{i k} \frac{\partial \delta A_{i}}{\partial x^{k}}\right) d V d t\right. \\
& =\int_{T} \int_{V}\left(j^{k} \delta A_{k}+f^{\prime}(\Delta)\right)\left(\mu_{0}^{-1} F^{i k} \frac{\partial \delta A_{k}}{\partial x^{i}}\right) d V d t
\end{aligned}
$$

Hence:

$$
0=\int_{T} \int_{V}\left(j^{k} \delta A_{k}+\frac{\partial f^{\prime}(\Delta) \mu_{0}^{-1} F^{i k} \delta A_{k}}{\partial x^{i}}-\frac{\partial f^{\prime}(\Delta) \mu_{0}^{-1} F^{i k}}{\partial x^{i}} \delta A_{k}\right) d V d t
$$

Using the Gauss theorem and taking into account the fact of the disappearance of the four-dimensional potential at the boundary of the four-dimensional space we obtain

$$
\frac{\partial f^{\prime}(\Delta) F^{i k}}{\partial x^{k}}=-\mu_{0} j^{i}
$$

Eqns. (9) include the second pair of Maxwell equations in the form eqns. (5) and (6). Whereas, on using the function $f(\cdot)$ as the identify function, its derivative will be equal to one; i.e.

$$
f^{\prime}\left(\frac{1}{4} \mu_{0}^{-1} F^{i k} F_{i k}\right)=f^{\prime}(\Delta)=1
$$


This means that the Maxwell equations in the form eqn. (9) include the classical Maxwell equations in the form of eqn. (5) and (6).

\section{INTERPRETATION OF THE ESTABLISHED EQUATIONS}

In three-dimensional notation, eqn. (9) has the following form:

$$
\operatorname{div}\left(\varepsilon_{0} f^{\prime}\left(\frac{1}{2} \mu_{0}^{-1} \boldsymbol{B}^{2}-\frac{1}{2} \varepsilon_{0} \boldsymbol{E}^{2}\right) \boldsymbol{E}\right)=\rho
$$

And

$$
\begin{aligned}
& \operatorname{curl}\left(v_{0} f^{\prime}\left(\frac{1}{2} \mu_{0}^{-1} \boldsymbol{B}^{2}-\frac{1}{2} \varepsilon_{0} \boldsymbol{E}^{2}\right) \boldsymbol{B}\right) \\
& =\boldsymbol{j}+\frac{\partial}{\partial t}\left(\varepsilon_{0} f^{\prime}\left(\frac{1}{2} \mu_{0}^{-1} \boldsymbol{B}^{2}-\frac{1}{2} \varepsilon_{0} \boldsymbol{E}^{2}\right) \boldsymbol{E}\right)
\end{aligned}
$$

The second pair of Maxwell equations could be rewritten in the same form as the well-known Maxwell equations (eqns. (5) and (6)):

$$
\operatorname{div}(\tilde{\varepsilon} \boldsymbol{E})=\rho \quad \operatorname{curl}(\tilde{v} \boldsymbol{B})=\boldsymbol{j}+\frac{\partial}{\partial t}(\tilde{\varepsilon} \boldsymbol{E})
$$

Where it was denoted:

$$
\begin{aligned}
& \tilde{\varepsilon}=\varepsilon_{0} f^{\prime}\left(\frac{1}{2} \mu_{0}^{-1} \boldsymbol{B}^{2}-\frac{1}{2} \varepsilon_{0} \boldsymbol{E}^{2}\right) \\
& \tilde{\mu}_{0}^{-1}=\mu_{0}^{-1} f^{\prime}\left(\frac{1}{2} \mu_{0}^{-1} \boldsymbol{B}^{2}-\frac{1}{2} \varepsilon_{0} \boldsymbol{E}^{2}\right)
\end{aligned}
$$

Lets us assume that the function $f(\cdot)$ can be almost linear or linear. Many experiments confirm that if the nonlinear character of the electromagnetic field equations exists, it cannot be strong; it must be weak. It seems to be reasonable to consider only the first nonlinear term of the Taylor series. Here we propose that:

$$
f(\cdot)=(\cdot)+\left(-k_{0}{ }^{2} T^{2}\right)(\cdot) \Rightarrow f^{\prime}(\cdot)=1+\kappa(\cdot)
$$

Thus:

$$
\begin{aligned}
& \tilde{\varepsilon}=\varepsilon_{0}\left(1+\kappa \mu_{0}^{-1} \boldsymbol{B}^{2}-\kappa \varepsilon_{0} \boldsymbol{E}^{2}\right) \\
& \tilde{\mu}_{0}^{-1}=\mu_{0}^{-1}\left(1+\kappa \mu_{0}^{-1} \boldsymbol{B}^{2}-\kappa \varepsilon_{0} \boldsymbol{E}^{2}\right)
\end{aligned}
$$

According to eqn. (11) and (12) the permittivity and reluctivity (or permeability) for free space have been changed with respect to the strong electric or magnetic field. The strong electromagnetic field causes a change of the free space coefficients. The multiplication ' $\varepsilon$ ' by ' $\mu$ ' is independent of the function $f(\cdot)$ and equal to $\varepsilon_{0} \mu_{0}$.

$$
\tilde{\varepsilon} \tilde{\mu}=\varepsilon_{0} \mu_{0}
$$

The Maxwell equations (in spite of their nonlinear character) still have the same form:

$$
\begin{array}{ll}
\operatorname{div}(\tilde{\varepsilon} \boldsymbol{E})=\rho & \operatorname{curl}\left(\tilde{\mu}_{0}^{-1} \boldsymbol{B}\right)=\boldsymbol{j}+\frac{\partial}{\partial t}(\tilde{\varepsilon} \boldsymbol{E}) \\
\operatorname{curl} \boldsymbol{E}=-\dot{\boldsymbol{B}} & \operatorname{div} \boldsymbol{B}=0
\end{array}
$$

The derivative $f^{\prime}(\cdot)$ which appears in the Maxwell equations is the reason for the nonlinear character of the generalised Maxwell equations with respect to electric field strength and magnetic flux density. The level of 'deformation' of the Maxwell equations in comparison with the linear Maxwell equations is determined by the constant ' $\kappa$ '. The less is the value of constant ' $\alpha$ ', the less is the influence of the nonlinear term in eqn. (12). We may evaluate (roughly) the value of this unknown constant.

With modern levels of measurement accuracy, we are able to use laboratory devices that enable determination of the value of magnetic flux density (or electric field strength) with very high accuracy $(0.01 \%)$, and the material parameters with the same relative error. In a magnetic field $B=2 T$ the variation of this material parameter will not be observed according to eqn. if:

$$
|\kappa|<\pi \times 10^{-11}\left[J^{-1}\right]
$$

The constant ' $\kappa$ ' is so small only in the case of strong magnetic or electric fields may the linear Maxwell equations deformation be observed and detected.

\section{GENERALIZATIONS OF MAXWELL THEORY FROM BORN-INFELD THEORY}

There are nonlinear electromagnetic field theories, e.g. Born-Infeld theory of the charged particle $[2,3]$. In this Born-Infeld theory the nonlinear Maxwell equations are obtained from the following action integral:

$$
I=\int_{T} \int_{V} b^{2}\left(1-\sqrt{1+\frac{c^{2}}{b^{2}} I_{1}-\frac{c^{2}}{b^{4}} I_{2}^{2}},\right) d V d t=\iint_{T} b_{V}^{2}(1-R) d V d t
$$

On this basis, the equations obtained are supposed to be valid inside the electric particle. For fields that are weak compared to the critical strength $b$, the Born- 
Infeld Lagrangian becomes the Lagrangian of classical Maxwell theory.

The well-known Born-Infeld Lagrangian is usually written as

$$
\mathrm{L}_{B I}=\frac{b^{2}}{\mu_{0} c^{2}}(1-R), \quad R=\sqrt{1+\frac{c^{2}}{b^{2}} I_{1}-\frac{c^{2}}{b^{4}} I_{2}^{2}},
$$

Where

$$
I_{1}=\boldsymbol{B}^{2}-\frac{1}{c^{2}} \boldsymbol{E}^{2}=\frac{1}{2} F_{i k} F^{i k}, I_{2}=\boldsymbol{B} \cdot \boldsymbol{E}=\frac{c}{4} F_{i k} F^{i k}, b \text { is a }
$$
maximum electric field strength (in the absence of magnetic field). If $b^{2}$ is very much larger than $\boldsymbol{E}^{2}$ and $c^{2} \boldsymbol{B}^{2}$, then $L_{B I} \approx-\left(1 / 2 \mu_{0}\right) I_{1}$ and we recover linear Maxwell theory. We remark here that in the limit as $c \rightarrow \infty, \mathrm{L}_{B I}$ tend to zero, while $c L_{B I}$ approach a well-defined, non-zero limit.

Since the Lagrangian density must be a Lorentz scalar, the electromagnetic field has only two gauge invariant Lorentz scalars, namely

$$
\begin{aligned}
F & =\frac{1}{2} F_{i k} F^{i k}=\boldsymbol{B}^{2}-\boldsymbol{E}^{2} \\
G^{2} & =\left(\frac{1}{4} F_{i k}^{*} F^{i k}\right)^{2}=(\boldsymbol{B} \cdot \boldsymbol{E})^{2}
\end{aligned}
$$

Where $F_{i k}^{*}=\frac{1}{2} \varepsilon^{i k \rho \sigma} F_{\rho \sigma}$ is the dual field strength tensor, Making $\mathrm{c}=1$ we have that equation (15) can be expressed as

$$
L_{B I}=a^{2}(1-R), \quad R=\sqrt{1+\frac{F}{a^{2}}-\frac{G^{2}}{a^{4}}},
$$

The field equations for Born-Infeld theory are

$$
\begin{aligned}
& \partial_{v}\left[\frac{\mathrm{F}^{i k}-{ }^{*} \mathrm{~F}^{i k} \mathrm{G} / \mathrm{a}^{2}}{\mathrm{R}}\right]=0, \\
& \partial_{j} F_{i k}+\partial_{k} F_{j i}+\partial_{i} F_{k j}=0
\end{aligned}
$$

Here, we find that the symmetric energy-momentum tensor for that theory is given by

$$
T^{i k}=\frac{F^{\mu j} F_{j}^{k}+G^{2} \eta^{i k} / a^{2}}{R}+\eta^{i k} a^{2} R
$$

In deriving this result, use has been made of the identity $F_{\mu v}^{*} F_{v \rho}=-G \delta_{\rho}^{v}$.
So the energy, the momentum, and the Poynting vector, are now given, respectively, by

$$
\varepsilon_{\text {field }}=\int d^{3} \vec{x}\left[\begin{array}{c}
\frac{\boldsymbol{E}^{2}+(\boldsymbol{E} \cdot \boldsymbol{B} / a)^{2}}{\sqrt{1+\frac{\boldsymbol{B}^{2}-\boldsymbol{E}^{2}}{a^{2}}-\frac{(\boldsymbol{E} \cdot \boldsymbol{B})^{2}}{a^{4}}}} \\
+a^{2}\left(\sqrt{1+\frac{\boldsymbol{B}^{2}-\boldsymbol{E}^{2}}{a^{2}}-\frac{(\boldsymbol{E} \cdot \boldsymbol{B})^{2}}{a^{4}}}\right)
\end{array}\right]
$$

$$
\boldsymbol{P}_{\text {field }}=\int d^{3} \vec{x} \frac{\boldsymbol{E} \times \boldsymbol{B}}{\sqrt{1+\frac{\boldsymbol{B}^{2}-\boldsymbol{E}^{2}}{a^{2}}-\frac{(\boldsymbol{E} \cdot \boldsymbol{B})^{2}}{a^{4}}}}
$$

$$
S=\frac{\boldsymbol{E} \times \boldsymbol{B}}{\sqrt{1+\frac{\boldsymbol{B}^{2}-\boldsymbol{E}^{2}}{a^{2}}-\frac{(\boldsymbol{E} \cdot \boldsymbol{B})^{2}}{a^{4}}}}
$$

\section{ENERGY TENSOR OF THE ELECTROMAGNETIC FIELD}

The volume density of the Lagrangian function in a region outside the electrical charges and currents is equal to

$$
f(\Delta)
$$

Where

$$
\begin{aligned}
\Delta=-\frac{v_{0}}{4} F_{\alpha \beta} F^{\alpha \beta} & =-\frac{v_{0}}{4} F_{\alpha \beta} F_{\gamma \delta} g^{\alpha \gamma} g^{\beta \delta} \\
& =a_{0} F_{\alpha \beta} F_{\lambda \gamma \delta} g^{\alpha \gamma} g^{\beta \delta}
\end{aligned}
$$

And $g^{i k}$ means the second-order metric tensor of space $[1,3,4]$.

Let us evaluate the energy tensor $T^{i k}$ by the definition $[1,5]$ in the following form:

$$
\frac{1}{2} \sqrt{-g} T_{i k}=\frac{\partial(f(\Delta) \sqrt{-g})}{\partial g^{i k}}-\frac{\partial}{\partial x^{l}}\left(\frac{\partial(f(\Delta) \sqrt{-g})}{\partial\left(\frac{g^{i k}}{\partial x^{l}}\right)}\right)
$$

The tensor satisfies the energy conservation law in the tour-dimensional form:

$$
T_{i, k}^{k}=0
$$


Substituting eqn. (20) into eqn. (26) one obtains:

$$
T_{i k}=\frac{2}{\sqrt{-g}} \frac{\partial(f(\Delta) \sqrt{-g})}{\partial g^{i k}}
$$

Because function $f(\Delta)$ is independent of the derivatives of the metric tensor.

Thus one obtains:

$$
T_{i k}=4 a_{0} \frac{\partial f}{\partial \Delta} F_{i}^{\delta} F_{k \delta}-g_{i k} f(\Delta)
$$

For the function $f(\cdot)$ given by the first two Taylor series terms we could write:

$$
f(\Delta)=\Delta+\kappa \Delta \quad \Rightarrow \quad f^{\prime}(\Delta)=1+\kappa
$$

Thus the energy tensor is equal to:

$$
T_{k}^{i}=\left.T_{k}^{i}\right|_{\kappa=0}+2 \kappa \Delta\left(\left.T_{k}^{i}\right|_{\kappa=0}+\delta_{k}^{i} \Delta\right)
$$

The trace of this energy tensor is equal to:

$$
T=T_{\alpha}^{\alpha}=\left.T_{\alpha}^{\alpha}\right|_{\kappa=0}+\kappa\left(\left.T_{\alpha}^{\alpha}\right|_{\kappa=0}+4 \Delta\right)=4 \kappa \Delta
$$

because, in the case $\kappa=0$, the trace of the covariantcontravariant energy tensor disappears:

$$
T_{\alpha}^{\alpha} \mathrm{I}_{\kappa=0}=4 a_{0} F^{i j} F_{i j}-\delta_{\alpha}^{\alpha} \Delta=0
$$

The trace of the energy tensor is not negative. The trace is equal to zero if and only if the constant ' $\kappa$ ' vanishes.

According to the main Einstein equations, for energy field for which one can introduce the energy tensor $[1,3$, 5] it could be written:

$$
R_{i k}-\frac{1}{2} g_{i k} R=-\frac{8 \pi G}{c^{2}} T_{i k}
$$

Contraction with respect to the indices ' $i$ ' and ' $k$ ' gives the Riemannian curvatura scalar of the electromagnetic field:

$$
R=-\frac{8 \pi G}{c^{2}} T
$$

Substituting eqn. (29) into eqn. (31) one finally obtains

$$
R=-\frac{32 \pi G}{c^{2}} \kappa \Delta
$$

The sign of constant $k$ thus, take into account an electrostatic field forced by one charged particle. Such a field has spherical symmetry. The Riemannian curvature scalar for two-dimensional space, where only one external charge is situated, must be non-negative, $R \geq 0$. Is not, the Riemannian curvature scalar tensor is negative, the space would have two radii of curvature, one positive and the other negative. This is impossible with respect to the assumed spherical symmetry of the electric field (forced by one electric charge), therefore:

$$
\kappa \leq 0
$$

In the case of a nonlinear electromagnetic field theories, e.g. Born-Infeld theory of the electromagnetic particle [5].

We can obtain an especial result. Und er a chiral approach, using equations $(21,22)$ with $\boldsymbol{E}=i \boldsymbol{B}$,we obtain $\boldsymbol{S}=0$ and an electromagnetic term which correspond to a cosmological constant given by $8 \pi G \varepsilon_{0} / c^{4}=1.8382 \cdot 10^{-54} \mathrm{Volt}^{-2}$. This allows the close connection between the electromagnetism and the gravitation (see annex).

\section{CONCLUSIONS}

Generalised Maxwell equations include the classical Maxwell equations of the electromagnetic field for weak fields. The reluctivity and permittivity of free space are changed. If the constant ' $\alpha$ ' cannot be omitted, the Riemannian-Christoffel curvature tensor is not equal to zero. The constant ' $\kappa$ ' is not positive: $\kappa \leq 0$. In the case of a nonlinear electromagnetic field theory, e.g. BornInfeld theory of the electromagnetic particle [5], we can obtain an especial result. Under a chiral approach, with $\boldsymbol{E}=i \boldsymbol{B}$, we obtain $\boldsymbol{S}=0$ and an electromagnetic term which correspond to a cosmological constant.

\section{ANNEX}

This work discovers the space-time curvature carried by the electromagnetic field and provides a new unification of geometry and classical electromagnetism. The new unification contains the Einstein equations to handle the mechanics and permits the derivation of the Maxwell equations from the full second Bianchi identities. This is a purely classical work and quantum considerations are merely mentioned. 
Central to this work are the requirements that the electromagnetic field be expressed as a two form $\mathrm{F}$ and fit into general relativity under the demand that the total stress-energy tensor used in the Einstein equations contain the Maxwell stress-energy tensor $T_{\text {Max }}$. In the notation with the conventions of [1] and in S.I. units $T_{M a x}$ is

$$
T_{\text {Max } k}^{i}=\frac{\varepsilon_{0}}{2}\left(F^{j i} F_{j k}+* F^{j i} * F_{j k}\right)
$$

where $\varepsilon_{0}=8.85418782 \cdot 10^{-12}$ farad $/$ meter is the electric vacuum permittivity.

Originally [2] general relativity was conceived as a unification of mechanics and geometry that explained gravitation. It was just a bonus [3] that electromagnetism also entered the unification via equation. If the Maxwell stress-energy tensor carried all the properties of the electromagnetic field, showing electromagnetism to be entirely reducible to mechanics, that would have been the end of the story.

However, the electromagnetic field has polarization or phase information that is not contained in the Maxwell stress-energy tensor [4]. Since Weyl's conformal tensor, the totally traceless piece of Riemann curvature, is supposed to contain the phase or polarization information carried by gravitational radiation, one should expect it to do the same for electromagnetic radiation.

This is born out by the discovery of a piece of the Weyl conformal tensor that depends explicitly on the electromagnetic field and contains this polarization or phase information. It is denoted by $\mathrm{T}_{\mathrm{Max}} C_{F}$, called "the local gravitational field of the electromagnetic field", and given by:

$$
\begin{aligned}
C_{j l}^{i k} & =8 \pi \frac{G \varepsilon_{0}}{c^{4}}\left\{\frac{3}{2}\left(F^{i k} F_{j l}-* F^{i k} * F_{j l}\right)-\frac{1}{4} \delta_{j l}^{i k} F^{i k} F_{i k}+\right. \\
& \left.+\frac{1}{4} \eta_{j l}^{i k} F^{i k} F_{i k}\right\}
\end{aligned}
$$

where $G=6.6726 \cdot 10^{-11}$ Newton-meter ${ }^{2} /$ kilogram $^{2}$ is Newton's gravitational constant, $c=2.99792458 \cdot 10^{8}$ meter/second is the speed of light, is a fully antisymmetric tensor. The traces in the expression for $C_{F}$ are the Lorentz invariants of the electromagnetic field $F^{i k} F_{i k}=-2\left(\boldsymbol{E}^{2}-\right.$ $\left.c^{2} \boldsymbol{B}^{2}\right)$ and ${ }^{*} F^{i k} F_{i k}=4 c(\boldsymbol{E} \cdot \boldsymbol{B})$, where $\boldsymbol{E}$ is the electric field strength in Volt/meter and $\boldsymbol{B}$ is the magnetic field strength in Tesla.
The major discovery of this work is the expression for $C_{F}$. The arguments that led to that expression are quite general and should defeat the criticism that $C_{F}$ was built on algebraically special black holes and will fail elsewhere. It would be useful to have a physical solution to the Einstein-Maxwell equations with non-zero currents that were not overwhelmed by symmetry. Then one could extend this analysis into the currents and see how the full second Bianchi identity works there. Further successful examples will give knowledge and comfort; but will not prove the generality for $C_{F}$ that is claimed here. However, a single credible counterexample or the observation of a magnetic monopole will vitiate this work.

The small coupling constant required by the Einstein equations, $8 \pi \frac{G \varepsilon_{0}}{c^{4}}=1.8382 \cdot 10^{-54} \operatorname{Volt}^{-2}$, permits the superposition of electromagnetic fields. It has also led many to believe that the gravitational consequences of electromagnetism are insignificant.

Nothing could be further from the truth. It is a matter of principle to unify classical electromagnetism and gravitation and the curvature-based unification presented here allows the electromagnetic field to appear as an algebraically special piece of curvature. This fulfills the nineteenth century speculation that gravity and electromagnetism are both aspects of Riemann curvature.

This theory is not experimentally vacuous. The smallness of the coupling constant merely means that it could be along time before curvature detectors are sufficiently sensitive while withstanding an intense electromagnetic field; or sufficiently sensitive over very long distances having less intense fields. One wonders about the consequences of $C_{F}$ in the environment around very strongly magnetised neutron stars [7]. Further, what are its consequences in the Jacobi equation for geodesic separation that might apply to trans galactic travel? When two electromagnetic fields are superposed could the interaction terms in the curvature have any bearing on the problem of emission or absorption?

The physical geometry of space-time is determined by specifying the metric tensor or the full curvature tensor [6]. The Einstein equations, which link classical mechanics to physical geometry, may be written as

$$
\begin{gathered}
M_{1 \gamma \delta}^{\alpha \beta}=\frac{8 \pi G}{c^{4}}\left(-\frac{1}{2} \delta_{\gamma \delta \rho}^{\alpha \beta \gamma}\left\{T_{\lambda}^{\rho}-\frac{1}{4} \delta_{\lambda}^{\rho} T\right\}\right) \\
\text { and } \\
M_{2 \gamma \delta}^{\alpha \beta}=\frac{8 \pi G}{c^{4}}\left(-\frac{1}{12} \delta_{\gamma \delta}^{\alpha \beta} T\right)
\end{gathered}
$$


where $\mathrm{T}$ is the total stress-energy tensor and $\mathrm{T}$ its trace. There is no mention of Weyl's conformal tensor that would complete the specification of the physical geometry.

Placing constraints on Weyl's conformal tensor is the novel feature of this work. Such constraints are meant to limit the solutions to those with a physical gravitational field. If the constraints are too limiting and they forbid physical solutions, then they will have to be altered. Similar constraints might deal with the embarrassing number of Ricci flat universes, which may or may not describe gravitational radiation. It is an open question whether the Einstein equations will have to be extended to the full curvature to handle gravitational radiation.

\section{REFERENCES}

[1] C. W. Misner, K. S. Thorne, and J.A. Wheeler. Gravitation. W. H. Freeman, San Francisco. 1973.
[2] A. Einstein. "The Principle of Relativity". Dover Publications, New York. 1952.

[3] D. Jackson. Classical Electrodynamics. $3^{\text {rd }}$ ed, pp. 273280, John Wiley \& Sons, New York. 1998

[4] T.T. Wu and C.N. Yang. Concept of Non integrable Phase Factors and Global Formulation of Gauge Fields. Phys. Rev. D. Vol. 12, pp. 3845-3857. 1975.

[5] H. Torres-Silva. "A new relativistic field theory of the electron". Ingeniare. Rev. chil. ing. Vol. 16 No 1, pp. 111-118. 2008.

[6] H. Torres-Silva. "Electrodinámica quiral: eslabón para la unificación del electromagnetismo y la gravitación”. Ingeniare. Rev. chil. ing. Vol. 16 $\mathrm{N}^{\circ}$ 1, pp. 6-23. 2008.

[7] W. E. Thirring. "An alternative approach to the theory of gravitation”. Ann. Phys. USA. Vol. 16, pp. 96-117. 1961. 